\title{
Dos décadas de estructuras plegadas de hormigón. Inicio y ocaso de un movimiento
}

\author{
Two decades of concrete folded plate construction. \\ Beginning and end of a movement
}

\author{
$\underline{\text { R. García }}{ }^{(*)}$
}

RESUMEN

En este trabajo se trata de la evolución y realizaciones más destacadas con estructuras formadas por plegaduras o facetas de hormigón armado. Se indagan los orígenes de su cálculo y aplicaciones y se ofrece una panorámica de lo realizado entre 1950 y 1970 . Estas dos décadas dieron lugar a una gran concentración de literatura técnica y publicaciones al respecto, para decaer rápidamente poco después. Singularmente, puede certificarse su final, al menos simbólico, con un destacado Congreso de la IASS celebrado en 1970 y dedicado expresamente a estructuras plegadas. Pasadas más de cuatro décadas desde las últimas grandes estructuras de este tipo, parecía pertinente realizar una revisión de sus logros y de las posibles causas de su decadencia. Esto es pertinente dado que, desde hace algunos años, se aprecia un renovado interés por las geometrías facetadas y de plegaduras.

\section{SUMMARY}

This paper deals with the development and achievements of structures conformed by concrete folded plates. It deals with the origins of its analysis and calculation and shows an overview of the main folded plate structures between 1950 and 1970. Great number of technical articles and publications on folded structures appeared in these two decades, to quickly decline shortly after. At least in a symbolic sense, their end can be certified by a memorable and monographic IASS symposium held in 1970 on such structures. More than four decades after the last of the large-scale structures of this type was erected, a review of achievements and possible causes of decadence seems to be pertinent, furthermore considering the growing and renewed interest in the geometry of folds and facets of the last years.
Palabras clave: Hormigón; láminas; plegadas; pliegues; estructuras.
Keywords: Concrete; plates; folded; folds; structures.

\footnotetext{
(*) Universidad Politécnica de Madrid, (España). 
1. Formas básicas de plegaduras prismáticas. Hanna, M.M. y Farad,

A.: "Optimum Dimensions.. Cast-In-Situ Prismatic Roofs"

2. Formas estructurales analizadas en artículos citados de Gruber, Fig. 1 y Fig.2; Ehlers, Abb.16, Abb.22 y Abb.26 y Craemer, Abb. 8 .

Folded plates en inglés, Flächentragwerke, Scheibenwerke o Faltwerke en alemán o toits pliseés en francés, en castellano no poseen una única traducción. En este trabajo hemos empleado para el título, de forma genérica, la denominación de láminas plegadas adoptada preferentemente por Cassinello (Hormigonería, ver referencia 30), aunque en algunos casos de espesores estructurales más importantes hemos usado también el término placa o losa plegada.

2 Este texto, adaptado y ampliado, tiene su origen en la conferencia del mismo título pronunciada el 11 de febrero de 2007 en la Fundación COAM de Madrid dentro del ciclo Historia de la Construcción dirigido por S. Huerta Fernández.

3 En él además menciona ser autor de las primeras construcciones de este tipo, correspondientes a los depósitos de carbón de la sala de calderas de la Märkische Elektrizitätswerk en Finkenheerd de 1924-25. Ofrece además cálculos para una cubierta simple a dos aguas a modo de generalización de la configuración inversa de los fondos de los depósitos.
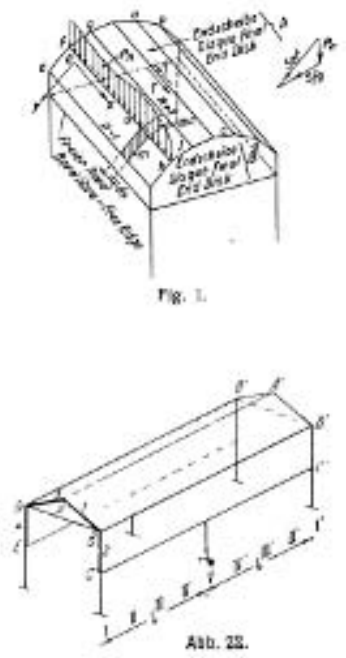

\section{INTRODUCCIÓN}

Las estructuras formadas por plegaduras delgadas de hormigón armado se diferencian de las de cáscara en el carácter plano de sus superficies, no siéndoles por tanto, de aplicación los beneficios de la curvatura ni el comportamiento pleno de membrana ${ }^{1}$. Según Angerer, "tienen la ventaja, frente a las cáscaras, de estar compuestas por superficies planas, lo que representa menores dificultades de encofrado" (1). Candela las definió como aquellas que, a diferencia el resto de estructuras laminares, están "sometidas a régimen mixto de esfuerzos de membrana y flexión" (2) (Figura 1).

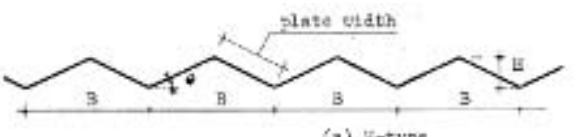
(a) V-typo
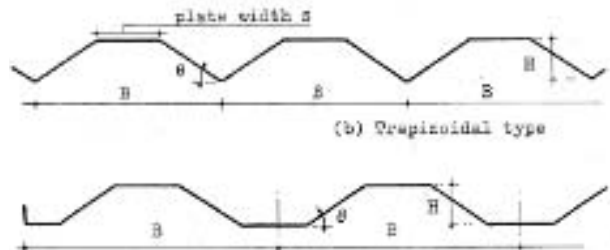

(e) 32at trapiasidal type YiE, 2 - kroup I - disgle forra of enlided plate roofe

La mayoría de ellas y sin duda las más importantes, tuvieron su momento álgido en las décadas de los 50 y los 60 del siglo XX. Singularmente, puede certificarse su final, al menos simbólico, con un destacado Congreso de la Internacional Asociation of Shell Structures (IASS) celebrado en 1970 y dedicado expresamente a este tipo de estructuras.

Pese a las esperanzas puestas en la geometría de plegaduras, alentadas por dicho Congreso, apenas se hicieron ya obras destacables a partir del mismo. Kimberly (Waggle) Kramer, estudioso de las mismas en EEUU ha sugerido para el corto pero fructífero periodo en que estuvieron en primer plano,
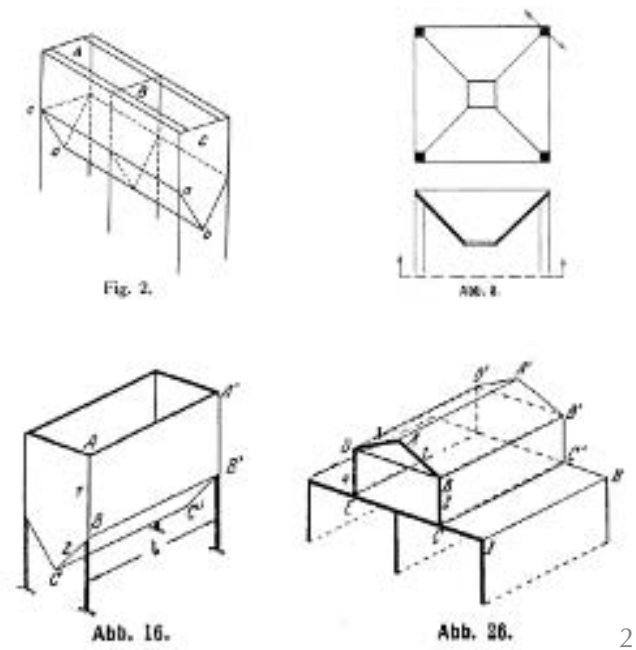

la denominación de "movimiento de las láminas plegadas" (3), un movimiento estructural, si es que así puede Ilamársele, cuyas realizaciones ocuparon muchísimas páginas de la literatura técnica y de las revistas de arquitectura, para después desvanecerse en silencio y rápidamente (4).

El objetivo de este artículo es una revisión arquitectónica e histórica de este tipo de estructuras, centrado en las realizaciones del periodo indicado más arriba. Es Ilamativo que, aunque dichas configuraciones estructurales en hormigón puedan considerarse un ciclo prácticamente cerrado, apenas se hayan estudiado en su conjunto ${ }^{2}$. Recientemente, sin embargo, se ha despertado un interés visible en estas formas como recursos de diseño arquitectónico. Ello ha venido acompañado de algunos trabajos parciales sobre el tema (5) (6) (7).

\section{LOS INICIOS}

C.B. Wilby en un resumen histórico sobre su análísis indica que: "El principio fue empleado por primera vez por Ehlers en Alemania en 1924, pero no para cubiertas sino para grandes depósitos de carbón, publicando un artículo sobre su análisis estructural en $1930^{\prime \prime}(8)^{3}$. Fueron estas estructuras de depósitos las que primero se construyeron, empleando placas plegadas en $\mathrm{V}$ para su fondo. Como a su vez corroboran Winter y Pei:

"Las primeras de tales estructuras, grandes depósitos de carbón, fueron erigidas en Alemania en 1925 y los primeros artículos sobre la teoría de su diseño se publicaron en ese país por G. Ehlers y H. Craemer en 1930. Desde entonces este tipo de construcción ha sido ampliamente usado en Europa y Rusia en un gran número de depósitos, cubiertas y otras estructuras... Durante unos años de trabajo como consultor en Rusia, el escritor senior de este artículo [Winter] tuvo ocasión de diseñar y ver erigirse una cierta cantidad de importantes estructuras de este tipo" (9).

No obstante, aparte de algunos dibujos no da más referencias concretas de dichas realizaciones.

Siguiendo nuevamente a Wilby:

"Después, en 1932, Gruber publicó un estudio en alemán. En los siguientes cinco años muchos europeos - Craemer, Ohlig, Girkman y Vlasov (1939) entre otros - hicieron contribuciones al respecto. Las teorías europeas eran generalmente complejas y arduas para su uso en el diseño. Desde 1945 se desarrollaron en EEUU métodos simplificados por Winter \& Pei 
(1947), Gaafar (1953), Simpson (1958), por Whitney (1959) adaptando el método de Girkmann, por Traum (1959), por Parme (1960) y por Goble (1964)" (10).

Candela considera también la aportación de Kazinsky (1948) (11) ${ }^{4}$ y A. Raymond cita como pionero a W. Flügge (12). Gruber aborda ya el caso de cubiertas prismáticas de sección poligonal (13) (Figura 2). Según Kramer "Aunque las estructuras de láminas plegadas de hormigón se introdujeron en los Estados Unidos en la primera parte de siglo XX, su desarrollo y empleo antes de 1950 fue infrecuente" (14).

En correspondencia con la extensión de métodos simplificados de cálculo, el ingeniero americano Milo Ketchum, autor de buen número de ellas, señaló como ventajas que:

"El análisis era directo, empleaba métodos a los que estaba acostumbrado y los elementos estructurales eran los que se usaban en otras estructuras de hormigón. [...] Es posible analizar las láminas plegadas con más precisión que las cáscaras cilíndricas" (15).

Ketchum contribuyó también al actual nombre en inglés de este tipo de estructuras:

"Siempre me disgustó el nombre de "cubrición a dos aguas (pitched roofs)", y cuando fui presidente de un comité ASCE (American Society of Civil Engineers), me hice responsable, al menos parcialmente, del cambio de nombre a lámina plegada (folded plate)" (15).

Pocas son las estructuras plegadas o prismáticas construidas de las que se tiene constancia antes de la segunda guerra mundial. El ejemplo más antiguo que conocemos antecede a los estudios teóricos citados y es la cubierta hexagonal de un depósito elevado de agua en Dubbeldam (Holanda) realizado por la empresa Stulemeyer en 1914. Su base tenía una separación entre lados de unos $9 \mathrm{~m}$ con una altura aproximada de $5,80 \mathrm{~m}$ y espesor de $8 \mathrm{~cm}$ (16) (Figura 3). Que sepamos es el ejemplo documentado más antiguo ilustrativo de la afirmación de Winter y Pei de que "además de dichas estructuras prismáticas, estructuras piramidales sin vigas, han encontrado aplicación en el continente para cubiertas de pabellón, torres de enfriamiento y otros propósitos" (17). No obstante, ambos autores publican en su trabajo dibujos de dos depósitos de carbón, uno de 1925 en Alemania, al que califican como primera estructura construida de dicho tipo, y otro, en Rusia, con placas de fondo inclinadas de algo más de $13 \mathrm{~m}$ de longitud (18).

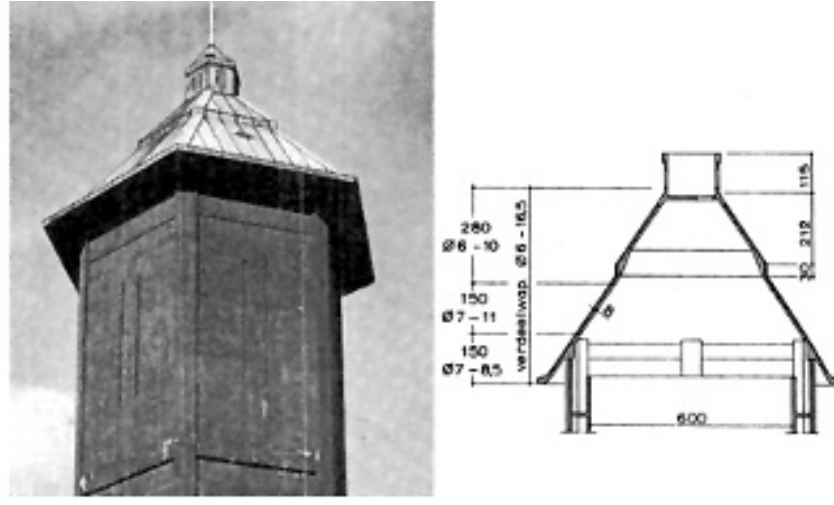

En EEUU, Winter y Pei cita como pionera una cubierta con tres faldones aligerados con casetones en San Francisco. Su autor fue L.H.Niskian y cubría un espacio de 44,33 pies de luz y 70 pies de largo. Consiguió un ahorro aproximado de 1,35 dólares $/ \mathrm{m}^{2}$ (de 1936) (19). Winter y Pei reflejaron también la información suministrada por el ingeniero A.H. Brownfield de que varias cubiertas de este y similares tipos se construyeron en California (18).

\section{LA ECLOSIÓN DE POSGUERRA}

Nuestra primera referencia de este periodo es un estudio de Ove Arup de 1946, en el que se plantea una superficie prismática para cubierta muy semejante a la que, a partir del año siguiente, también ocuparía la atención de Félix Candela (20). No obstante, y en cuanto a realizaciones, la figura adelantada parece ser Candela, autor en México de las primeras estructuras de plegaduras modernas de que se tiene constancia. Su éxito posterior con los paraboloides hiperbólicos dejó un tanto oscurecidas estas experiencias y la mayoría de las monografías actuales pasan por ellas sin concederles apenas importancia. Su aludido estudio de 1947 se refería a naves y cubiertas industriales con láminas de sección en Z y en él, además de explicar los métodos de cálculo y sus limitaciones incluía detalles muy precisos de armado y unas perspectivas muy elocuentes (21) (Figura 4).

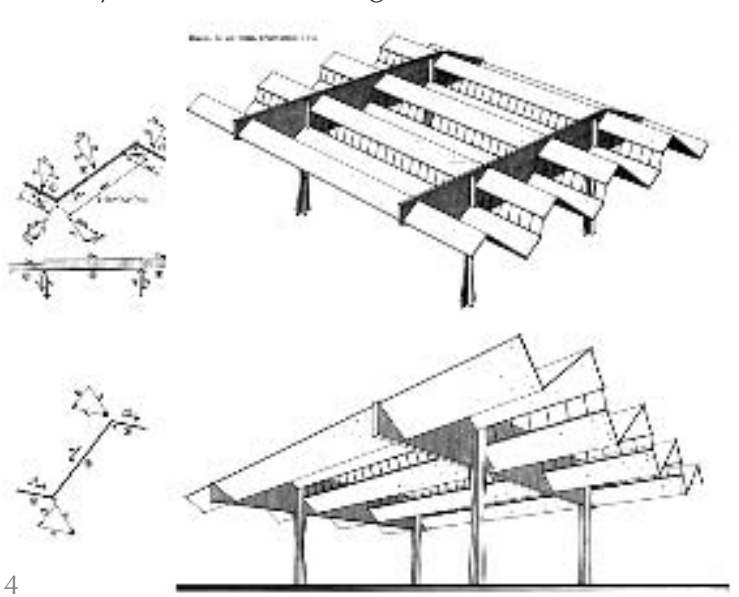

3. Depósito elevado en Dubbeldam (Países Bajos). Cement, (1961), p. 440 y bunker ruso. Winter y Pei: "Hipped Plate Construction" (1947), p. 521.

4. Estudio de cubiertas prismáticas para usos industriales. Félix Candela, 1947. Faber. Félix Candela, p. 69.
4 En este trabajo se introdujo el método del análisis límite. 
5. Almacén y talleres H.W. Moore Equipment Co., Denver. M. Ketchum, 1955. "Folded plate shell concrete roofs". Architectural Forum, (Feb. 1955), p. 156
Entre 1951 y 1953 Candela construyó al menos cinco estructuras plegadas, pero no del tipo anterior, sino del tipo de cumbreras y valles planos, prosiguiendo con otras realizaciones hasta 1957. La que parece ser la primera es la cubierta de la casa del número dos de la calle Novedades de ciudad de México, que fue seguida de un garaje en la misma ciudad con vuelos de 3 m (22). En dos de sus siguientes proyectos las utilizara no sólo como formas de cubrición, sino también como estructuras de entrepisos. Esto se pudo ver en la Escuela Montes Alpes de dos plantas y en un seminario con cuatro pisos, que originariamente iban a ser seis, ambos también en Ciudad de México (23). Sin embargo, posteriormente reconoció que para pisos era un "camino de investigación sin esperanzas" (23). Para cubiertas, no obstante, el sistema parecía competitivo ya que en 1957 volvió a emplearlas en un supermercado (24). Faber indica que, además: "La losa plegada aparece de nuevo en una o dos pequeñas fábricas, pero ahora en forma de dientes de sierra, como en el techo de la capilla de La MedaIla Milagrosa" (24).

En EEUU se produjo justo también en los primeros años cincuenta una auténtica eclosión de estas construcciones. Una figura de referencia es el ya mencionado ingeniero Milo Ketchum. Ketchum reconoce en sus memorias que fue influido por Candela y refiere además su primer encuentro con el arquitecto español (15).

Respecto al papel que en su popularización, sobre todo en EEUU, tuvo la puesta a punto de métodos simplificados de cálculo ha de destacarse que fue en 1947 cuando se publicó el célebre trabajo de Winter y Pei, ampliamente citado y que además abrió el camino a multitud de trabajos posteriores buscando métodos más accesibles. Este trabajo es tenido en cuenta por Candela al hablar de sus propias láminas, aunque también advierte que fuera de los casos más sencillos, sus simplificaciones podrían conducir a fallos importantes (21).

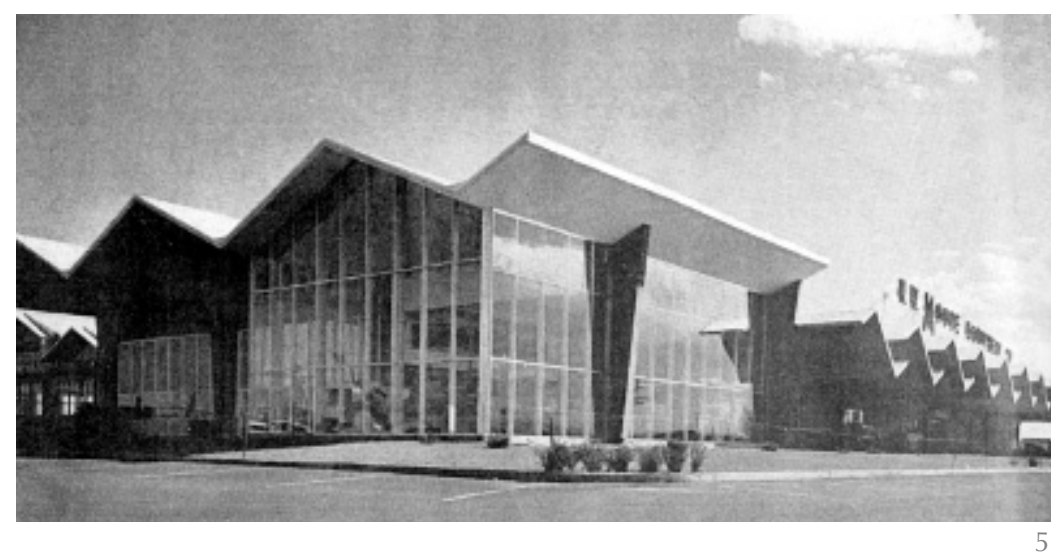

Como última alusión al cálculo, diremos que muy poco antes, en 1945, el trabajo de Lundgren sugirió (indirectamente porque se refería a láminas curvadas cilíndricas) otro método alternativo al de Winter y Pei y basado en el efecto viga, sobre todo en estructuras de canto grande y proporciones alargadas (25). Esta idea fue explícitamente expresada por el profesor Cassinello:

"Las primeras soluciones plegadas [...] nacen por similitud con las losas onduladas o láminas cilíndricas. La idea que se desarrolla es muy simple: aumentando el brazo de palanca de la estructura, se puede salvar mayor luz sin aumentar mucho el peso; los cordones superior e inferior de cada plano inclinado alojan las armaduras principales, mientras que a lo largo de su faldón se absorben los esfuerzos cortantes" (26).

Volviendo a la difusión americana, la memoria de Ketchum ofrece los tipos de estructuras de cubiertas plegadas más típicas. En muchas, especialmente las de losa plegada continua, son comunes los vuelos extremos que las hacen parecer más gráciles y aéreas y que, además, eran elementos necesarios para evitar la inestabilidad del borde libre.

Como realizaciones de Ketchum destaca el almacén y talleres de maquinaria de construcción H.W. Moore Equipment Co, cerca de Denver (1955) (27) (28) (Figura 5). Es Ilamativo que se optara por este sistema debido al ahorro en la póliza contra incendio, ya que al ser de hormigón, compensaba el superior coste respecto a otros sistemas habituales. Se usaron diferentes tipos de perfiles plegados para las naves, con espesores oscilando entre 4 y 6 pulgadas para luces de hasta $24 \mathrm{~m}$ en sentido longitudinal. Un puente grúa con cargas de hasta 15 to colgaba de las naves de mayor altura.

Otras obras realizadas y citadas por el mismo Ketchum son la Casey Junior High School en Boulder, el gimnasio de la St Mary's High School y la Arvada High School sin referencia de localizaciones para estas últimas. También cita dos iglesias, una de ellas de planta circular y otra en Minnesota, esta última con placas triangulares (15). Así mismo, y entre las primeras obras de cierta envergadura con láminas plegadas en EEUU, estarían las cubiertas para la Langendorf Bakery de Los Angeles (1955), obtenidas por la repetición de un módulo cuadrado en planta de aproximadamente $18 \mathrm{~m}$ de lado, pero empleando ya técnicas de pretensado. Esta lámina puede incluirse en lo que Ketchum Ilamó el "three segments type" (perfil trapecial) (29) (30). 
Como muestra el caso anterior, a partir de la mitad de la década de los cincuenta las plegaduras de hormigón comenzaron a beneficiarse de las técnicas del pre y postensado, ganándose en esbelteces y luces alcanzadas. Con postensados fueron notables ejemplos, también en EEUU, las instalaciones de la Cloverleaf Line Bowling Alley en Dade County con 36,6 m de vano (1958) (3) y la que seguramente fue la de mayor luz de su época, el gimnasio del Indiana State Teachers College con 50,3 m (1961) (31), ambas con sección trapecial y hormigonadas in situ. El postensado hará posible además una nueva opción: el montaje por placas separadas cosidas posteriormente mediante cables.

\section{PREFABRICACIÓN}

Una de las aplicaciones más extendidas del pretensado en placas plegadas fue la elaboración de prefabricados. El elemento base mayoritariamente utilizado fue la placa de perfil trapezoidal con ligeras prolongaciones, la cual satisfacía el ideal teórico de un único tipo de elemento para toda la construcción. Para el ingeniero suizo Tirhamer L. Koncz:

"deberían tener un espesor mínimo de 2 $1 / 2$ pulgadas... la placa plegada debería fabricarse sobre un encofrado deslizante, lo cual solo es posible con inclinación de paredes no mayor de $45^{\circ}$. El espesor es determinado por su posible transporte... los elementos pueden fabricarse sobre una larga línea de producción....En este caso los cables pueden pretensarse para varios elementos a la vez" $y$, en cuanto a los encofrados, "deberían ser de hormigón ya que si no, al vibrarse el hormigón desliza por las paredes inclinadas... La velocidad es de aproximadamente 5 pies por minuto" (32).

Un sistema tan sencillo dio lugar a múltiples variantes en función, por ejemplo, de las soluciones de iluminación adoptadas, lo que fue especialmente adecuado en usos industriales. En el artículo de Konz se ilustran ejemplos desarrollados por él mismo en Alemania (contratista L. Rostan, Friedichshafen), en Cesena (Italia) o en Florencia. El mismo autor muestra también una interesante experiencia de empleo como losas de forjados y otra en la que las placas se usan a la vez como cerramientos y cubiertas, ambas construidas también en Italia. Con igual autoría se ilustra además el sistema desarrollado en Suiza para optimizar la luz norte y basado en lucernarios inclinados a $60^{\circ}$, muy cercanos al ángulo óptimo de iluminación. Se trataba de placas en Z, "las primeras construidas mediante pretensado" (Figura 6).
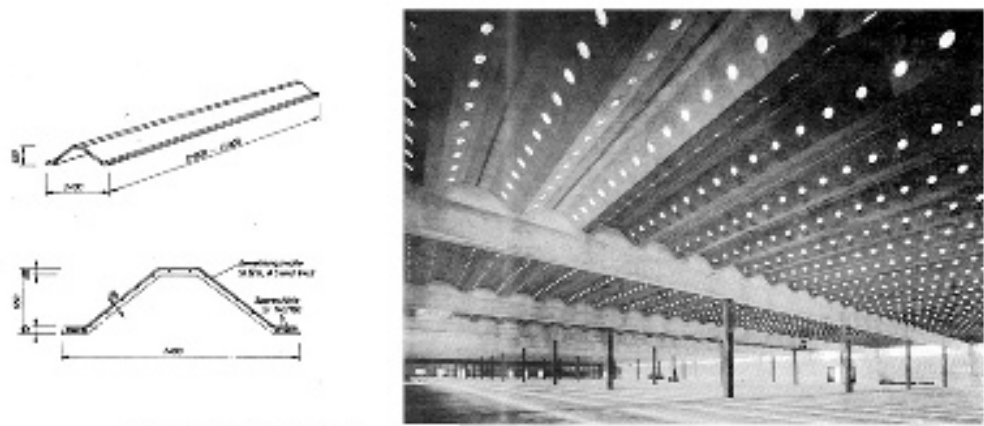

NORTH LIGHT'ROOF

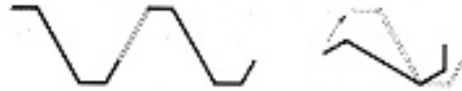

Estos sistemas fueron de gran rendimiento "cubriendo cada placa una superficie de hasta 1000 pies cuadrados. Para su colocación se precisan 4 ó 5 hombres y cada elemento necesita entre 8 y 10 minutos" (32).

De prefabricados trapezoidales W. C. Harry ofrece la referencia en EEUU de un elemento que, aunque "originalmente diseñado para un encargo concreto, sus unidades encontraron un uso creciente y llegaron a ser un producto estándar en Florida" (33). Según describe, la idea se inició en 1958 por un arquitecto y un ingeniero que "trabajando en estrecha colaboración con un fabricante de prefabricados de hormigón" (33) deseaban emplear grandes unidades de este tipo plegado para cubiertas "en un área en donde el prefabricado era popular" (33). Ese primer encargo fue la escuela elemental Avocado en Homestead, Florida con vanos de 50 pies en el comedor y 30 pies en las aulas, estas últimas con voladizos exteriores.

Harry diseñó dicha escuela así como otras cuatro edificaciones descritas en su artículo y que incluían una vivienda, un hangar con un vuelo de más de 15 m (Mackey Airlines), una iglesia (Pompano Beach) y un muro para un estadio de baseball. Cada uno de los proyectos presenta una solución singular e imaginativa del uso de dichas láminas plegadas.

Estos sistemas prefabricados tuvieron también amplia aceptación en los países del Este, en la órbita de la antigua Unión Soviética, al considerarse especialmente adecuados para los fines de una economía planificada. La antigua DDR los desarrolló, al parecer, masivamente con plantas de gran producción (34) y en el caso de Rumania merece citarse un sistema muy particular de pretensado para placas descrito por A. Mihul (35), que disponía los cables oblicuamente. Típico de este sistema fue la fabricación de placas planas que se unían después en obra adoptando formas plega-
6. Almacén, Cesena, Italia y sistemas de iluminación. Koncz, Tilhamer L.: "Prestressed Precast Folded Plates for Industrial Buildings". IASS Symposium, vol. 1, (Vienna: 1970), Fig. 6. 
das normalmente de perfil sencillo de picos y valles. Con ellas se realizó en Rumania una singular central térmica en Roman de planta circular. Otras aplicaciones notables en el mismo país, fueron los pabellones de deportes de Piatra Neamt y de la villa Gh. Gheorghiu-Dej (Figura 7).
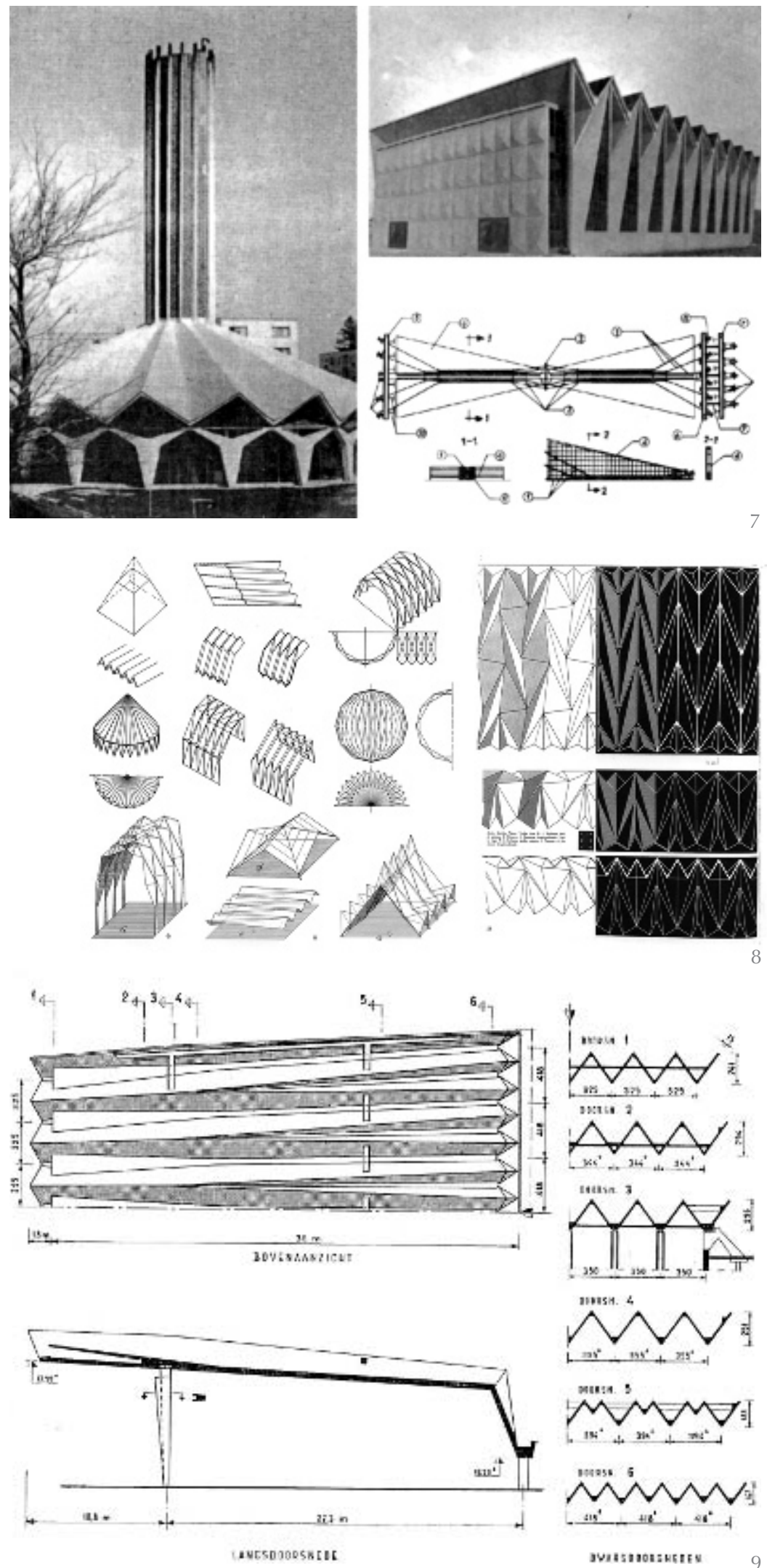

\section{GEOMETRÍA}

Tras las soluciones prismáticas más simples, pronto pudo verse un aumento en la variedad de formas construidas. Repertorios de configuraciones posibles han sido recogidos por Engel y Angerer (36) (37). También es notable el adelantado trabajo del arquitecto italiano Guido Figus con sus complejas formas plegadas publicadas como posibles soluciones de cubrición de un auditorio (38). Pero que sepamos, no se han hecho intentos hasta ahora de contrastar dichas configuraciones especulativas con lo efectivamente realizado (Figura 8). También se debería de diferenciar entre plegaduras reales, en las que estructura y forma constituyen una unidad, y aparentes. Un ejemplo muy ilustrativo de lo segundo es la capilla de la Academia del Aire en Colorado Springs, realizada por SOM y cuya forma exterior plegada está en realidad construida con entramados metálicos (39). Resumimos a continuación las principales configuraciones encontradas en ejemplos construidos.

\subsection{Formas afiladas o con conicidad (indicadas para voladizos)}

Se obtienen por el deseo de variar el canto y así adaptarse mejor a la demanda de los momentos flectores. Un sistema sencillo para satisfacerla y no recogido en los trabajos mencionados (Engel, Angerer), fue duplicar los pliegues haciendo de cada uno inicial dos más pequeños de la mitad de canto intercalando nuevas aristas de doblez inclinadas. Dos casos representativos son los voladizos del pabellón de actividades de tiempo libre en la Wasserwerkstrasse de Zurich y en la singular cubierta del mercado de subastas de pescado en Scheveningen, Países Bajos (40) (Figura 9). El mismo efecto se puede conseguir también transformando la lámina desde un perfil en $\checkmark$ a otro en U Ilegando en el límite al borde plano, solución denominada por Angerer "plegadura con conicidad" (41). Esta idea se siguió en el aeropuerto de Fiumicino en Roma del ingeniero Morandi y arquitectos Luccichenti, Monaco y Zavitteri (42), y en la original tribuna con vuelos de $18 \mathrm{~m}$ del canódromo de Madrid de los arquitectos J.R. Azpiazu y P. Pinto, con intervención de los ingenieros J.A. Torroja, F. del Pozo y R. López Palanco (43) ${ }^{5}$.

No obstante, las láminas plegadas en vuelo más espectaculares de las que tenemos noticia fueron dos hangares construidos en 1958; en Miami para la National Airlines y en Utica (New York) para la Mohawk Airlines. El ejemplo de Miami tenía dos cubiertas en voladizo de 110 pies a ambos lados de una sección central para oficinas de 
dos pisos (Figura 10). Semejante solución, incluso con vuelos mayores, se había empleado ya, pero con cables de suspensión, primero en un hangar para la Trans-World Airlines en Kansas City y luego en dos ejemplos posteriores (44).

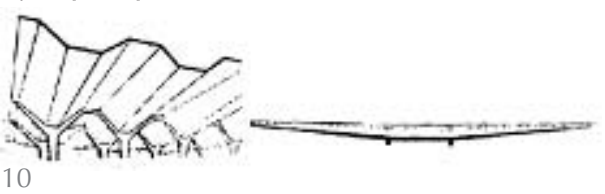

\subsection{Pliegues contrapuestos}

Una solución al problema de un rápido desagüe para estas estructuras está en el sistema de pliegues alternados o contrapuestos, en el que las cumbreras son horizontales pero oblicuas en planta. Un ejemplo es la cubierta diseñada por De la Vega y E. Torroja para los comedores de la Universidad Laboral de Tarragona, España (45). También el pabellón IBM de la Exposición Internacional de Bruselas de 1958 de Eliot Noyes (46) y el hipódromo de Groenendaal (Bélgica) de los años setenta por CERAU Architects (47), muestran soluciones similares de pliegues alternados, aunque con las limas hoyas inversamente dispuestas. Engel proporciona seis variantes de plegaduras contrapuestas, la mitad de las cuales no son desarrollables sobre un plano.

\subsection{Sistemas "aporticados"}

Entre las nuevas aplicaciones sucesivamente aparecidas está el sistema denominado por Angerer "aporticado" o de plegaduras aporticadas, obtenido haciendo continuar las láminas de cubierta con los cerramientos, que también se convertían en elementos resistentes (48). Con ello se conseguía, además de una mayor integración estructural, una unidad completa entre forma arquitectónica y estructura. Un ejemplo destacado es la sala mortuoria del cementerio de FrancfurtWesthausen, del arquitecto Günther Bock (49). Aquí el pórtico es asimétrico con apoyo en soportes de horquillas en un lado y muros plegados en el otro. Su cubierta es de plegaduras alternadas semejante a la realizada por E. Torroja (Figura 11).
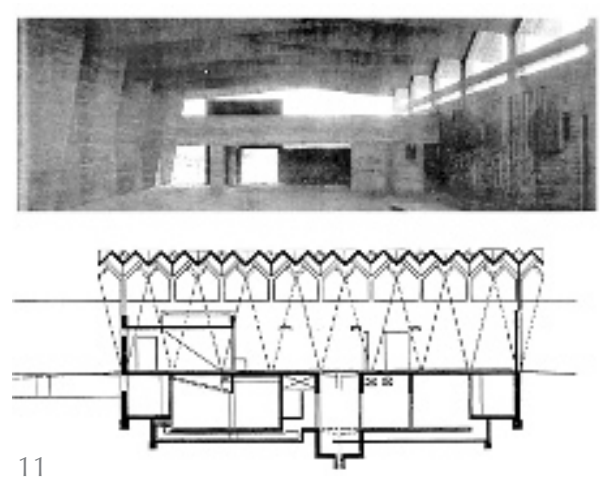

Del sistema "aporticado" una variante relativamente extendida es la formada por láminas con elementos triangulares que se asimilarían a la idea de pórtico con tres articulaciones, justamente en los puntos más afilados. Los triángulos vacíos se utilizaron generalmente para lucernarios en el techo o para accesos laterales y acristalamientos triangulares en las paredes. Un ejemplo es el pabellón deportivo de un liceo técnico en Boulogne sur Mer del arquitecto Pierre Dufétel y los ingenieros René Sarger y André Bloc (50) (Figura 12). Esta misma configuración encontró fácil aplicación adaptándose a plantas circulares, como en la St. AlbertKirche en Freiburg (Alemania) diseñada por E. Heine (1967-68) (51) y en el Hampton Coliseum en Virginia (1970), aunque en éste complementándose con otra estructura central no plegada de cubierta.

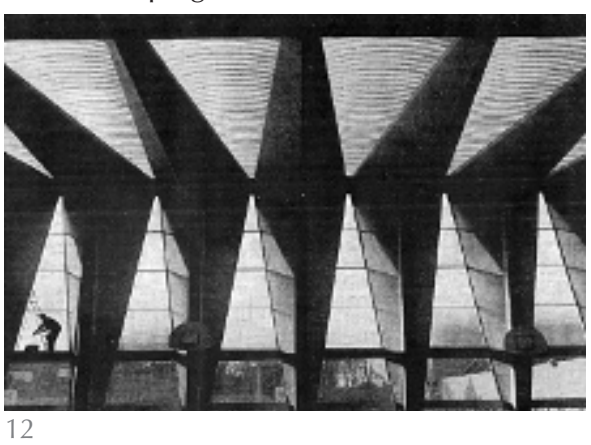

\subsection{Abovedados}

Una singular forma asimilable a este tipo se obtuvo en un conjunto de piscinas y gimnasios cubiertos en Copenhague, calculados por el ingeniero Eric Ingerslev (52). Aunque con ciertas semejanzas con ejemplos anteriores, los triángulos no formaron pórticos sencillos, sino configuraciones facetadas cóncavo-convexas de tipo poliédrico y no de base prismática, con un esquema característicamente no desarrollable (Figura 13).

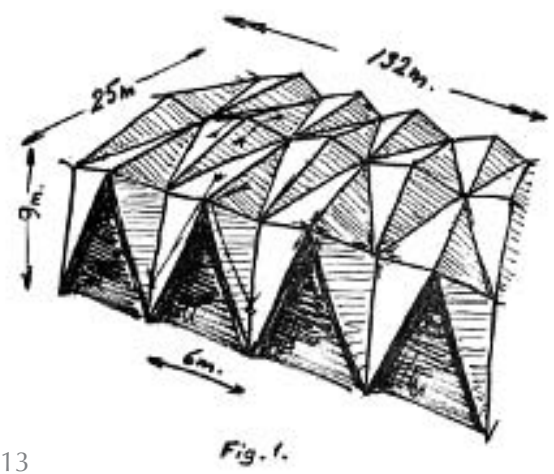

No obstante, la forma más típica para abovedamientos se obtiene mediante pliegues cóncavo-convexos que, en su forma más característica, constituyen, en cambio, una superficie desarrollable ${ }^{6}$. De esta disposición son especialmente sugestivas las aulas de la Universidad Politécnica de Budapest
7. Rumania. Central térmica en Roman, centro deportivo en Gh. Gheorghiu-Dej y pretensado simultáneo de cuatro placas triangulares. Mihul, Anatolie.: "Prismatic Roofs of Prestressed Concrete" $^{\prime \prime}$ IASS Symposium, vol. 2 (Vienna: 1970), pp. 3, 4 y 20.

8. Tipos de plegaduras. Angerer, Construcción laminar, 1972, pp. 46, 51, 75-77, Engel, Sistemas de estructuras, 2000, pp. 222, 226-228 y Guido Figus, "Structura come forma", Spazio, vol.3, no. $6,1951-52$, p. 28.

9. Lonja de Scheveningen (Países Bajos). Seyn, W.K. y Hofman, J.W.: "De constructie van het visafslaggebouw te Scheveningen". Cement, no. 8 (1964), p. 466

10. Hangar para la National Airlines, Miami, 1958. "Folded Plates Roof New Hangars". Architectural Record, no. 3 (Mar. 1958), p. 223

11. Sala mortuoria cementerio de Francfurt-Westhausen. Günther Bock, arquitecto. Wieschemann, Paul Gerhard y Gatz, Konrad. Edificios de hormigón, 1969 , p. 159.

12. Liceo técnico, Boulogne sur Mer. Pierre Dufétel, arquitecto, y René Sarger y André Bloc, ingenieros. Wieschemann, Paul Gerhard y Gatz, Konrad. Edificios de hormigón, 1969, p. 140.

13. "Sport Stadium near Copenhagen", E.Ingerslev, IASS Symposium, vol. 2, (Viena 1970), fig.1.

5 Similar al sistema con conicidad es el de pliegues no paralelos, en la que éstos, tanto con perfiles en $U$ como en $\mathrm{V}$, reducen progresivamente su sección transversal y convergen hacia un punto. Esta disposición fue empleada principalmente en plantas con formas convergentes o trapeciales. Dos notables ejemplos son las cubiertas de la estación Moscú de metro en Budapest y de la estación de tren de Den Helder en los Países Bajos.

${ }^{6}$ Esta forma corresponde al pliegue Yoshimura del origami. 
14. Instituto alemán de la industria del cemento, Düsseldorf. E. y P. Neufert, arquitectos. Kanstein, F.: "Nieuw duits onderzoekinstituut voor de cementindustrie". Cement, no. 19-20 (1956) p. 473.

15. Aula, TU Delft. Van den Broek y Bakema, arquitectos, 1965. H van Dusschotten "Het auditorium voor de Technische Hogeschool te Delft (IV). De constructie van het dak" Cement no. 5 , (1964), p. 270.
7 Son notables también las estructuras de diferentes pabellones del arquitecto Carlos Raul Villanueva en la Universidad de Caracas. A ellas puede sumarse la tribuna del estadio de Maracaibo. Debo a la arquitecta venezolana Mónica Silva Contreras las referencias de ejemplos de Caracas.

${ }^{8}$ La formación de facetas es característica del tipo poliédrico frente a las placas alargadas del tipo prismático. Angerer, Construcción laminar, p. 49 considera también el tipo semiprismático como una derivación de las formas piramidales.
(53) y las cubiertas del teleférico del Ávila en Caracas, obra esta última del arquitecto Alejandro Pietri con el ingeniero Juancho Otaola (54). En Venezuela se encuentran también otros ejemplos de plegaduras de hormigón de gran atractivo (55) (56) ${ }^{7}$.

\section{EXPRESIVIDAD}

Por su novedad, las plegaduras se emplearon también intencionalmente como signos de modernidad y distinción. Algunas asociaciones e instituciones de ingeniería dedicados al hormigón las utilizaron como elementos representativos en sus propios edificios. Lo vemos en la nueva sede del Instituto alemán de investigación de la industria del cemento en Dusseldorf proyectado por E. y P. Neufert (1956), con una lámina plegada bien visible como cubierta del ático (57) (58). El edificio posee además una elegante marquesina de entrada, que repite el esquema de cubierta (Figura 14). Otro ejemplo es el pabellón sede del American Concrete Institute (1958) con cubierta de dos series de láminas en voladizo. Fue su autor Minoru Yamasaky, en esta época muy interesado en el sistema (59) (60). También incluimos el depósito de carbón en forma de dodecaedro de hormigón del instituto Eduardo Torroja de Madrid, inscrito en una esfera de $10 \mathrm{~m}$ de diámetro. Éste ilustra además el tipo plegado poliédrico, una variante dentro del sistema general de láminas plegadas, diferente del grupo más extendido de las de tipo prismático. Formas en artesa o pirámide truncada como la cubierta del hipódromo realizado entre 1959-60 por los arquitectos Gabetti e Isola en Nichelino cerca de Turín pueden incluirse en esta categoría (5) ${ }^{8}$.

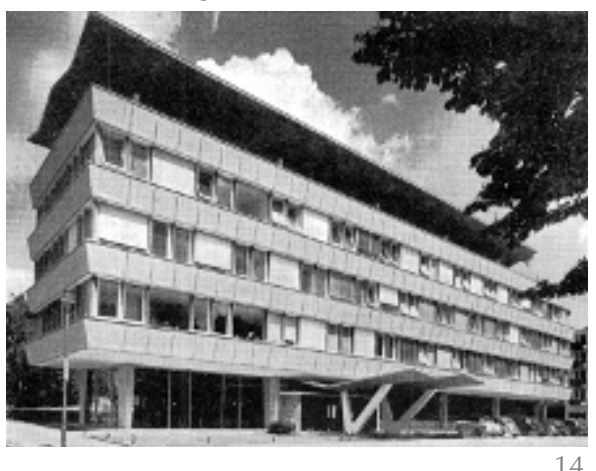

A su vez, varios otros arquitectos de renombre emplearon, a menudo como recurso expresivo, este tipo de estructuras. CassineIlo indicó que no fueron solo las complejidades de cálculo las que frenaron su uso, "sino los prejuicios estéticos ante una nueva forma que iba a romper con las doctrinas y moldes en boga" (61). En su opinión fueron factores favorables para su aceptación,

"el triunfo espectacular de la arquitectura agresiva, personal y dinámica de Wright, y el ensayo, en gran escala, desarrollado en la estructuralista Exposición de Bruselas... la lámina plegada se impone y marca la transición de la ortogonalidad a la curva; de la placa a la bóveda; del estatismo al dinamismo propio de una estética estructuralista y sincera" (61).

Los arquitectos holandeses Bakema y Van den Broek realizaron con plegaduras y facetas la estructura del suelo y las cubiertas del aula de la TUDelft (1965), logrando un voladizo récord de $32 \mathrm{~m}$ (6) (Figura 15). También emplearon un sistema de pliegues porticados de gran luz en el ayuntamiento de Marl (62). Marcel Breuer proyectó una serie de unidades piramidales invertidas en las oficinas principales de la Van Leer, en Amstelveen (1959) (63) (64) y es suyo también otro interesante ejemplo de formas porticadas en la abadía de St John, Minnesota (1961). Algo antes, Pier Luigi Nervi había realizado, junto con Breuer y Bernard Zehrfuss, la cubierta de mayor luz realizada con láminas plegadas en un edificio no utilitario, en la sede de la UNESCO en París (1958). Así mismo, Louis Kahn también optó, aunque algo más tarde, por una lámina plegada cóncava cruciforme en la Iglesia Unitaria de Rochester (1967), el mismo año de la propuesta de Jørn Utzon y Richard Weston para el estadio de Jeddah en Arabia Saudí.

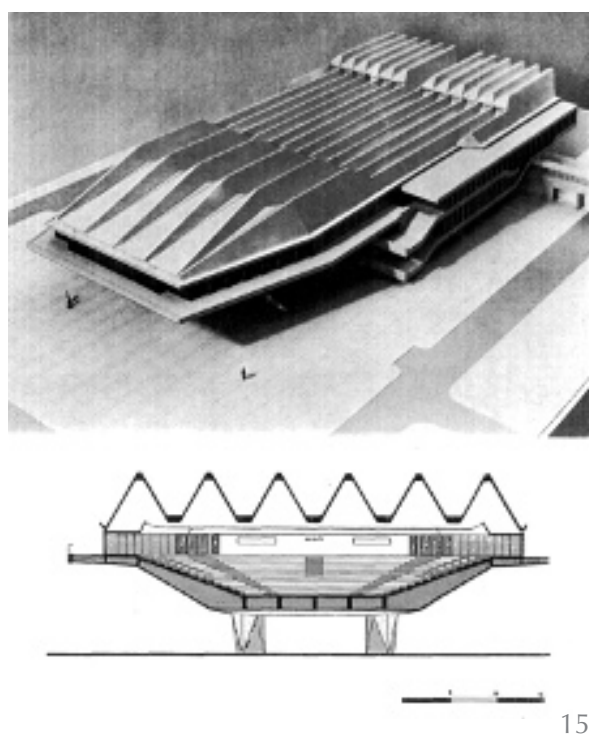

Como caso especial, el arquitecto alemán Gottfried Böhm experimentó con formas plegadas al menos desde 1957. En su trayectoria sobresale por su complejidad la capilla de peregrinaje de María, Reina de la Paz, en Velbert-Neviges, Bergisches land, terminada en 1972 con proyecto de 1963 (65). En ella, la cubierta se desarrolla en forma de un conjunto de pirámides irregulares interpenetradas y ejecutadas como rigurosas láminas de hormigón. También en Alemania es digna de mención la iglesia de St. Josef en Neuß-Weckhoven del arquitec- 
to H. Schaller con los ingenieros S. Polóny y R. v. Kalmar (1967) (66). Ésta se puede describir como una evolución sometida a deformaciones del tema básico de la bóveda de plegaduras (Figura 16).
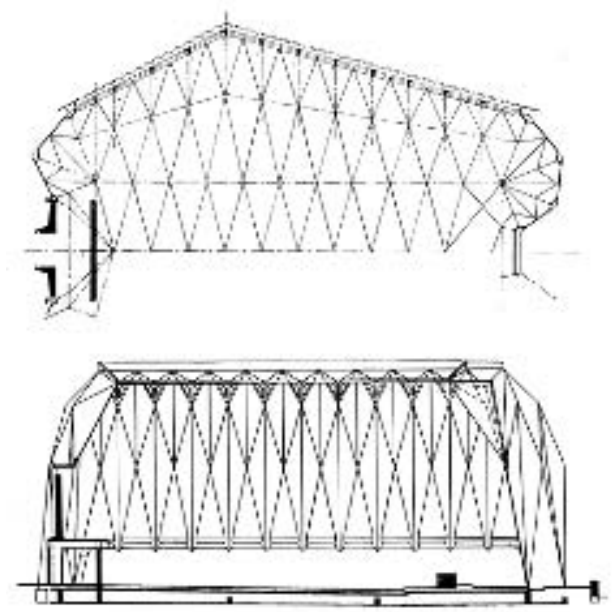

Finalmente, un recorrido de realizaciones merecedoras de comentario nos llevaría a Japón en donde, además de algunos ejemplos interesantes en arquitectura industria como una factoría para la Can Company en Tokio del arquitecto Ichiro Ebeihbara y una fábrica diseñada por RIA Group (67) (68) son destacables los proyectos de Kunio Maekawa para la Asamblea pública de Setagaya y para el City Hall de Imabari y sobre todo, el teatro del centro musical de la prefectura de Gunma, Takasaki, obra de Antonin Raymond (1961) (69) (70). Dicho teatro se basó en plegaduras simples en $\mathrm{V}$ pero adaptadas transversalmente a la forma trapecial de la planta, lo que conllevó un especial trazado de directrices quebradas y un desarrollo descendente de los pliegues para adaptarse a la convergencia de la sala (Figura 17).

\section{CELEBRACIÓN Y CLAUSURA}

En vista de las realizaciones Ilevadas a cabo hasta entonces, el Symposium Internacional de Viena de 1970 sobre Láminas Plegadas y Estructuras Prismáticas se clausuró con grandes expectativas. En él se puso de manifiesto, no sólo el estado de la cuestión, sino también las múltiples líneas de potencial desarrollo futuro para estas estructuras. En los nueve puntos de las conclusiones se indicaba, entre otras cosas:

- Que se habían desarrollado teorías completamente exactas para su cálculo, pero aún se debería trabajar en métodos aproximados más sencillos para su diseño, especialmente si se hacían intervenir materiales de distinta composición.

- Que era esta cuestión de los nuevos materiales y su empleo, a veces conjunto con el hormigón, la que más prometedora se veía en cuanto a comportamiento y economía.

- Que debía profundizarse en el conocimiento del comportamiento en rotura y que para ello serían muy útiles los modelos a gran escala.

- Que aspectos como la prefabricación, particularmente con hormigón ligero, deberían atraer más estudios, así como la protección contra el fuego.

- Y, finalmente, se exhortaba a "Arquitectos e ingenieros a trabajar juntos para crear nuevas construcciones que satisficieran estética, funcional y estructuralmente los requerimientos de nuestro tiempo" (71).

Entre los múltiples campos abordados en el congreso destacamos primeramente, los estudios centrados en la geometría de los pliegues. A ellos pertenece, por ejemplo, el de K. Miura, sobre una categoría de láminas denominadas por su autor PCCP SHELLS (Pseudo-Cylindrical Concave-Polyhedral Shells), obtenidas como derivación de superficies cilíndricas lisas transformadas a semejanza del aplastamiento de un cilindro de pared delgada (72). Sobre ese mismo tema, la lámina plegada cinemática de R. Resch y H.N. Christiansen, permitía adoptar todo tipo de formas abovedadas e incluso formas tubulares deformables a voluntad (73).

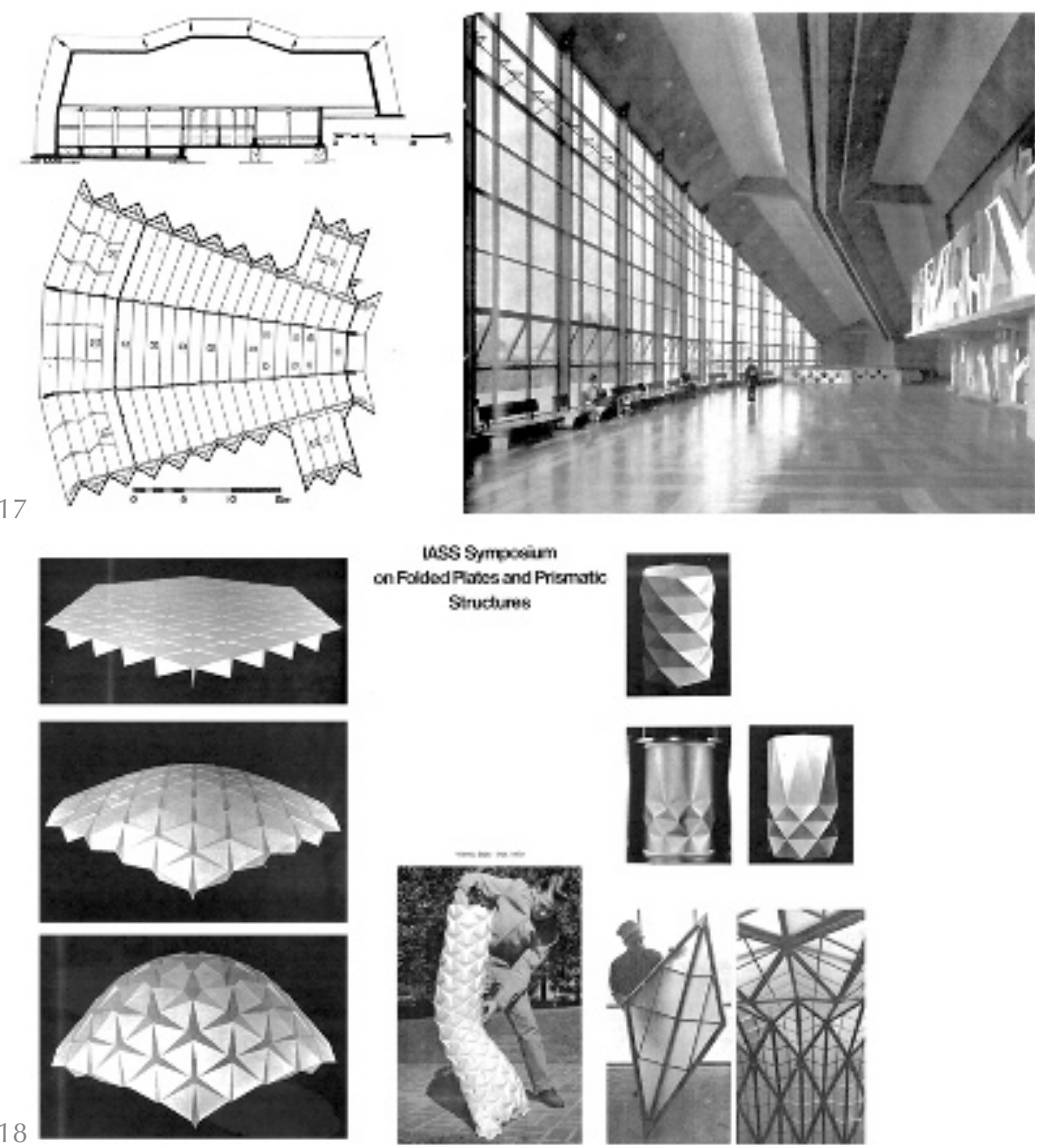

16. Iglelsia de St Paulus, antes St Joseph, Neuß-Weckhoven (Alemania). H. Schaller, arquitecto y S. Polóny y R.v. Kalmar, ingenieros, 1967. "Schalen und Faltwerke", Bauwelt 58, no 36 (4 September 1967), p. 912.

17. Centro musical Gunma, Takasaki (Japón). Antonin Raymond, arquitecto, 1961. Informes de la Construcción $n^{\circ} 144$, (1962).

18. IASS Symposium on Folded Plate and Prismatic Structures. Arriba derecha, K. Miura, PCCP (Pseudo-Cylindrical ConcavePolyhedral); abajo izquierda, R. Resch y H.N. Christensen, sistema de plegaduras cinemáticas; abajo derecha, A. Kolozsvary, lámina plegada universal. IASS Symposium, vols. 1, 2, Vienna, 1970. 
19. Hangar Alleghany Airlines, aeropuerto Logan, Boston, 1970. Th.A. Amsler, "Alleghany Airlines Hangar, Logan International Airport, Boston, Mass., U.S.A.", IASS Symposium, vol. 2, Vienna, 1970.

${ }^{9}$ Véanse artículos de Tihamer L. Koncz, K. Weißbach, E. Kühn y Anatolie Mihul en referencias bibliográficas.

${ }^{10}$ Un posterior IASS Symposium on Folded Plates and Spatial Panel Structures celebrado en Udine en 1974 ya mostró para éstas una clara decadencia. Pese a las todavía relevantes aportaciones teóricas y de métodos de cálculo, las únicas estructuras construidas dignas de mención se redujeron prácticamente a dos ejemplos, la estación Kursky de Moscú y la ya mencionada marquesina de la estación de metro Moscú en Budapest (nota a pie 5), con vanos de 27 y $24,5 \mathrm{~m}$ y voladizos de 12 y $9,5 \mathrm{~m}$ respectivamente.
Estas investigaciones especulativas estuvieron muy ligadas a nuevos materiales plásticos, como la propuesta de "Lámina plegada universal" (UFP) de A. Kolozsvary, basada en un rombo plegado estándar, repetido las veces necesarias (74), o la de P. Huybers del laboratorio Stevin de estructuras de Delft y que se apoyaba también en la construcción de un prototipo pero basado en una única pieza triangular unida al resto mediante solapes por pestañas salientes (75) (Figura 18).

Respecto a métodos de cálculo, se abordaron tanto nuevas teorías y métodos generales, como análisis de modelos a escala y estudios del comportamiento de materiales nuevos y compuestos. Un interesante apartado lo constituyeron los sistemas con pequeños plegamientos, algunos tipo sandwich. Con ellos se podían formar superficies alabeadas, como las del hangar para la American Airlines en Los Ángeles, creando voladizos en paraboloide hiperbólico de 230 pies basados en una simple chapa de acero plegada (76).

Un número significativo de ponencias giró en torno a la prefabricación de láminas plegadas con un extenso panorama de lo realizado en Suiza, Alemania Democrática y Rumania ${ }^{9}$. A destacar también fue el trabajo publicado por dos ingenieros de El Cairo (M.M. Hanna y A. Farad) sobre los límites razonables y económicos de estas estructuras dentro de configuraciones convencionales (77) (Figura 1).

Como culminación de realizaciones se presentaron las dos estructuras plegadas de hormigón armado de mayor magnitud
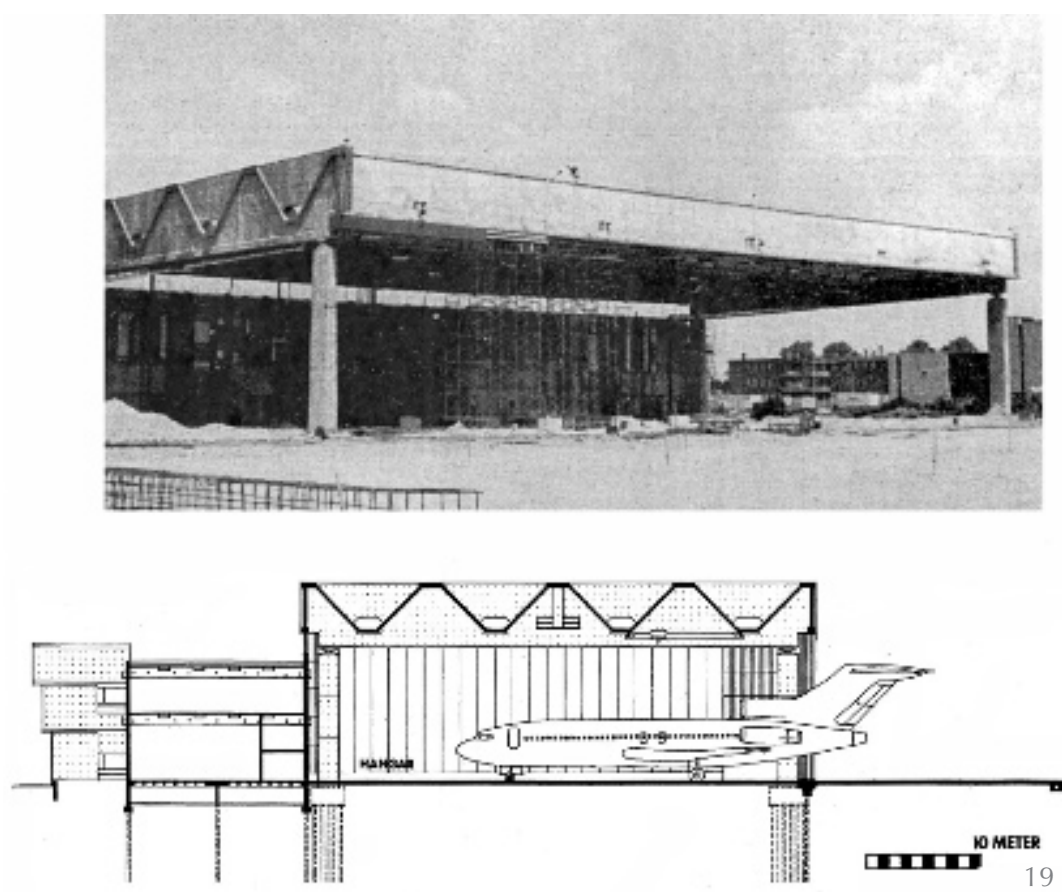

realizadas hasta la fecha. Una, la ya mencionada y relativamente temprana sala de conferencias de la sede de la UNESCO (78) en París (1958) y la otra un gran hangar para la Alleghany Airlines en el aeropuerto Logan, Boston (1970) que, con una luz longitudinal de 252 pies, excedió en 100 pies la mayor estructura de su clase construida anteriormente (79) (Figura 19).

\section{CONCLUSIONES}

Esta breve exposición de contenidos refleja el estado de la cuestión y las esperanzas puestas en un sistema que, según Wilby: "permite cubiertas de gran durabilidad, grandes luces con hormigón armado, líneas nítidas y estéticamente satisfactorias para muchos arquitectos" (80). Sin embargo, a dicho symposium le sucedió en realidad un marcado cambio de trayectoria que prácticamente conllevó al ocaso de dos décadas de intenso trabajo y experimentación sobre este tipo de estructuras ${ }^{10}$. Pero lo fue también para las plegaduras con otros materiales ya que, en construcción civil y arquitectura, no mucho más se ha realizado con ellos. Una pérdida de competitividad por encarecimiento de costos, así como las limitaciones inherentes de sus luces son las causas que normalmente suelen citarse como determinantes en el rápido abandono. Kramer agrega, al menos en EEUU, "la falta de comprensión de las estructuras plegadas por ingenieros y arquitectos" aunque considera prometedora la irrupción de los métodos de análisis por ordenador (4), siendo cierto, así mismo, que aportaciones puntuales sobre métodos de cálculo no han dejado de aparecer hasta el presente. Pero puede que también, tras más de veinte años de investigación se llegara a sentir un cierto agotamiento de sus posibilidades creativas y expresivas. En referencia a ello Milos Ketchum escribió: "Alguna vez en el futuro, el costo del acero estructural subirá por encima de lo previsible y alguien descubrirá la utilidad y belleza de las estructuras laminares, las diseñará, encontrará que son vendibles, las hará publicidad y el círculo de su popularidad comenzará de nuevo. Las cosas no se construyen o hacen por ser económicas, bellas o utilitarias. Se construyen o hacen porque alguien desea construirlas o hacerlas, y en el proceso se convierten en económicas, bellas o utilitarias" (81).

Pasadas más de cuatro décadas desde las últimas grandes estructuras de este tipo parecía pertinente realizar una revisión de sus logros y aspiraciones e intentar cubrir un evidente vacío en la historia reciente de las formas constructivas y arquitectónicas. Es un hecho que desde hace algunos años existe un renovado interés por las geome- 
trías facetadas y de plegaduras. No corresponde aquí analizar la situación actual, pero sí sugerimos que el inicio de tal revitalización parece haberse producido en los primeros años del nuevo siglo. El proyecto de iglesia parroquial de Nuestra Señora de la Asunción en Port Coquitlam, Bristish Columbia (Canadá) por Patkau architects (82), o la terminal de Yokohama de A. Zaera, pueden ser singulares testimonios del inicio de dicha revitalización. Sin embargo, tras estas propuestas, en cierto modo continuadoras del periodo clásico aquí considerado, el interés actual parece haberse desplazado hacia el ámbito formal, abandonando o al menos relajando, la más íntima relación entre forma y estructura que caracterizó aquellas realizaciones.

\section{REFERENCIAS}

(1) Angerer, Fred. (1972). Construcción laminar. Elementos y estructuración. 51, Gustavo Gili, Barcelona. (Bauten mit tragenden flächen konstruktion und gestaltung, 1960).

(2) Faber, Colin. (1970). Las estructuras de Félix Candela. p. 23, Compañía editorial continental, México, España, Argentina, Chile (Candela, Shell Builder, 1963).

(3) Kramer, Kimberly Waggle. (2005). Survey of Prestressed / Post-Tensioned Folded Plate Shells for Roof Structures in the United States: 1950-1970. Ned H. Burns Symposium on Historical Innovations in Prestressed Concrete, eds. Bruce W. Russell and Shawn P. Gross (Michigan: American Concrete Institute-SP 231), p. 70.

(4) Kramer, Kimberly Waggle. (2005). Survey of Prestressed / Post-Tensioned Folded Plate Shells for Roof Structures in the United States: 1950-1970. Ned H. Burns Symposium on Historical Innovations in Prestressed Concrete, eds. Bruce W. Russell and Shawn P. Gross (Michigan: American Concrete Institute-SP 231), p. 76.

(5) Staufer, A., Bonomo, R. (2006). Lichtraumfaltwerk. Steildachexperimente in den 50er un 60er Jahren. Werk, Bauen+Wohnen, (4): 28-35.

(6) García, R. (2006). Concrete folded plate structures in the Netherlands. Proceedings of the Second International Congress of History of the Construction, Cambridge, pp. 1189-1208

(7) Láminas plegadas de hormigón armado. Realizaciones en España, Actas del V Congreso Nacional de Historia de la Construcción. (2007). Burgos, pp. 367-378.

(8) Wilby, C. B. (1998). Concrete folded plate roofs. Arnold, London, p. 20. El artículo citado es: G. Ehlers. (1930). Die Spannungsmittlung in Flächentragwerken. Beton und Eisen 29, pp. 281-286, 291-296.

(9) Winter, George y Pei, Minglung. (1947). Hipped Plate Construction. Journal of the American Concrete Institute, 18(5): 505-506. El artículo citado de Craemer es: Craemer, H. (1930). Allgemeine Theorie der Faltwerke. Beton und Eisen, (29): 276-81.

(10) Wilby, C. B. (1998). Concrete folded plate roofs. Arnold, London, pp. 20, 23 y 24.

(11) G. de Kazinsky. (1948). Die Berechnung der Faltwerke nach dem Traglastverfahren. Proceedings 3rd Congress, IABSE, Liege, pp. 615-621.

(12) Raymond, A. y Rado, L. L. (1962). Centro musical Gunma. Informes de la construcción, (144), sin paginar.

(13) Gruber, E. (1932). Berechnung prismatisher Scheibenwerke. Memoires, Association Internationale des Ponts et Charpentes, (1): 225-241.

(14) Kramer, Kimberly Waggle. (2005). Survey of Prestressed / Post-Tensioned Folded Plate Shells for Roof Structures in the United States: 1950-1970. Ned H. Burns Symposium on Historical Innovations in Prestressed Concrete, eds. Bruce W. Russell and Shawn P. Gross (Michigan: American Concrete Institute-SP 231), p. 66.

(15) Ketchum, M. Memoirs 4. Folded Plates, (c. 1990), www.ketchum.org/shellpix. html (visitado 30 dic. 2009)

(16) Overzicht Nederlandsche schaalconstructies. Cement, (1961), p. 440.

(17) Winter, George y Pei, Minglung. (1947). Hipped Plate Construction. Journal of the American Concrete Institute, 18(5): 522.

(18) Winter, George y Pei, Minglung. (1947). Hipped Plate Construction. Journal of the American Concrete Institute, 18(5): 519-521.

(19) Concrete Roof of Novel Design for San Francisco Warehouse. Engineering News-Record, (1936), p. 558.

(20) Arup, Ove N.: Shell Construction. Yearbook for 1946-Architecture in Ireland, Dublin, Royal Institute of the Architects of Ireland.

(21) Faber, Colin. (1970). Las estructuras de Félix Candela. Compañía editorial continental, México, España, Argentina, Chile (Candela, Shell Builder, 1963). pp. 69-73.

(22) Agencia alquiler automóviles Nash, Anzures, D.F. con F. Fernández, (demolida). Faber. Félix Candela, p.64.

(23) Faber, Colin. (1970). Las estructuras de Félix Candela. Compañía editorial continental, México, España, Argentina, Chile (Candela, Shell Builder, 1963). pp.65, 67. 
(24) Faber, Colin. (1970). Las estructuras de Félix Candela. Compañía editorial continental, México, España, Argentina, Chile (Candela, Shell Builder, 1963). p. 68.

(25) Lundgren, H. 1945). Stabiliteitsforsøg med cylindriske jernbetonskaller. Ingeniørvidenskabelige skrifter, n. ${ }^{\circ}$ 5, Copenhage. Publicado después en Lundgren, H. (1951). Cylindrical shells, Danish Technical Press, Copenhagen.

(26) Cassinello, F. (1974 ). Construcción: Hormigonería. Ed. Rueda, Madrid, p. 537.

(27) Ketchum, Milo S. (1955). Design and Construction of a Folded Plate Roof Structure. Journal of the American Concrete Institute, 26(5): 449-455.

(28) Folded plate shell concrete roofs. Architectural Forum, (1955), pp. 156-57, en donde además de a Winter y Pei como referencias para los cálculos cita también a Ibrahim Gaasfar, Hipped Plate Analysis, Considering Joint Displacements, ASCE, Vol. 79, (April 1953).

(29) Kramer. Folded Plate Shells, p. 69

(30) Driskell, John J. (1957). Multispan Prestressed Folded Plate Roof for Langendorf Bakery, Journal of the American Concrete Institute, 29(2): 115-125.

(31) Prestressed Folded Plate Roofs a Gym. Architectural Record (junio, 1961), pp. 172-74.

(32) Koncz, Tihamer L. (1970). Prestressed Precast Folded Plates for Industrial Buildings. IASS Symposium, vol. 1, pp. 3, 6 (orden correlativo, no paginado)

(33) Harry, Walter C. (1963). Precast Folded Plates Become Standard Products. Journal of the American Concrete Institute 60(10): 1375.

(34) Weißbach, K. (1970). Manufacturing of VT-Folds y Kühn, E. Construction and Application of VT-Folds, ambos en IASS Symposium, vol.2, Vienna.

(35) Mihul, A. (1970). Prismatic Roofs of Prestressed Concrete. IASS Symposium, vol. 2, Vienna.

(36) Engel, H. (2000). Sistemas de estructuras. Gustavo Gili, Barcelona (ed. original alemán 1967), pp. 219-29

(37) Angerer, Fred. (1972). Construcción laminar. Elementos y estructuración. Gustavo Gili, Barcelona. pp. 41-77.

(38) Publicadas como ilustraciones sin apenas comentarios en Luigi Moretti. (1951-52). Struttura come forma. Spazio, 3(6): 21-30.

(39) Air Academy chapel shapes up. Architectural Forum, n. 114 (May 1961), pp. 128-29.

(40) Seyn, W.K. y Hofman, J.W. (1964). De constructie van het visafslaggebouw te Scheveningen. Cement, (8): 461-67.

(41) Angerer, Fred. (1972). Construcción laminar. Elementos y estructuración. Gustavo Gili, Barcelona. pp. 78-79.

(42) Fiumicino. (1961). Aeropuerto internacional de Roma. Informes de la construcción, n. ${ }^{\circ} 36$, sin paginar.

(43) Azpiazu, J. R., et al. (1962). Canódromo madrileño. Informes de la Construcción, n. ${ }^{\circ} 138$, sin paginar.

(44) Folded Plates Roof New Hangars. Architectural Record, n. ${ }^{\circ} 3$ (1958), pp. 223-27.

(45) Torroja, E. (1959). Lámina plegada. Universidad Laboral de Tarragona. Informes de la Construcción, n. ${ }^{\circ} 107$, sin paginar.

(46) IBM online digital archives, http://www-03.ibm.com/ibm/history/exhibits/vintage/ vintage_4506VV2259.html, (visitada 10 enero 2010).

(47) Ishikawa Lab, Album of Spatial Structures (ALOSS) http://www.anc-d.fukui-u. ac.jp/ ishikawa/Aloss/data/folded_plate/Catalog/catalog1.htm (visitada 10 enero 2010).

(48) Angerer, Fred. (1972). Construcción laminar. Elementos y estructuración. Gustavo Gili, Barcelona. pp. 74-75.

(49) Wieschemann, Paul Gerhard y Gatz, Konrad. (1969). Edificios de hormigón, Gustavo Gili, Barcelona, p. 159.

(50) Wieschemann, Paul Gerhard y Gatz, Konrad. (1969). Edificios de hormigón, Gustavo Gili, Barcelona, p. 140.

(51) Bologna, G. ed. (1974). Die St. Albert-Kirche in Freiburg (BR Deutschland). Lichtbeton im Hoch-und Ingenieurbau, Cembureau, Paris, pp. 180-185.

(52) Ingerslev, E. (1970). Sports Stadium near Copenhagen. IASS Symposium, vol. 2 (Vienna).

(53) ALOSS, http://www.anc-d.fukui-u.ac.jp/ ishikawa/years/unknown/Structural\%20Systems-Countries/etc/Europe/19xx-folded-HUNGARY/Hall..,TechUnivofbudapest.htm (visitada 12 enero 2010)

(54) Hernández de Lasala, S. (1995). El Teleférico de el Avila. Alejandro Pietri Arquitecto, pp. 88-109, Fundación Andrés Sosa Pietri, Caracas.

(55) Moholy-Nagy, S. (1999). Carlos Raúl Villanueva y la arquitectura de Venezuela. Instituto del Patrimonio Cultural, Caracas.

(56) Hernández de Lasala, S. (2006). En busca de lo sublime: Villanueva y la Ciudad Universitaria de Caracas. Consejo de Preservación y Desarrollo, Universidad Central de Venezuela, Caracas.

(57) Kanstein, F. (1956). Nieuw duits onderzoek-instituut voor de cementindustrie. Cement, (19-20): 473. 
(58) Künzel, W. (1956). Het uiterlijk aanzien van beton. Cement, (23-24): 572-573.

(59) Whitney, Charles S. (1958). Cantilevered folded plate roofs ACI headquarters. Journal of the American Concrete Institute, 30(4): 427-430.

(60) Strang, J. (1958). Construction for ACl. Journal of the American Concrete Institute 30(4): 431-38.

(61) Casinello, F. (1961). Estructuras plegadas. Informes de la Construcción, n. ${ }^{\circ}$ 135, sin paginar.

(62) Wieschemann, Paul Gerhard y Gatz, Konrad. (1969). Edificios de hormigón, Gustavo Gili, Barcelona, p. 157.

(63) Kantoorgebouw Van Leer's Vatenfabrieken N.V. te Amstelveen. Bouwkundig Weekblad, no. 48 (1959) p. 574

(64) Van der Werf, A. (1958-59). Kantoorgebouw te Amstelveen. Technisch Bouwblad, n. ${ }^{\circ} 21$, pp. 333-36.

(65) Pehnt, Wolfgang. (1999) Gottfried Böhm. pp. 30, 58-59, 64-66, 75-79, Birkhauser: Basel, Berlin, Boston.

(66) Schalen und Faltwerke, Bauwelt. (1967). (58)36: 912.

(67) Henn, W. (1965). Buildings for Industry. Hyden, Iliffe books, New York, London, pp. 93-95.

(68) Kenchiku bunka, n. ${ }^{\circ} 145$ (1958) pp. 49-51.

(69) A Folded Plate Shell for Concerts and Kabuki. Architectural Record. (1962). vol. 132, pp. 157-162.

(70) Centro musical Gunma. Takasaki, Japón. Informes de la Construcción, n. ${ }^{\circ}$ 144, (1962) pp. 146-63.

(71) Beer, H. (1970). Conclusions. IASS Symposium, vol. 2, Vienna.

(72) Miura, K. (1970). Proposition of Pseudo-Cylindrical Concave Polyhedral Shell. IASS Symposium, vol. 1, Vienna.

(73) Resch, R. y Christiansen, H.N. (1970). Kinematic Folded Plate System. IASS Symposium, vol. 1, Vienna.

(74) Kolozsvary, A. (1970). The Universal Folded Plate (UFP) Industrial Structural System. IASS Symposium, vol. 2, Vienna: .

(75) Huybers, P. (1970). Large Span Folded Structures in Plastics. IASS Symposium, vol. 2, Vienna.

(76) Zetlin, L. y Thornton, Ch.H. (1970). World's Larges Light Gage Steel Folded Hyperbolic Paraboloidal Shell Roof Structure. IASS Symposium, vol. 2, Vienna.

(77) Hanna, M.M. y Farad, A. (1970). Optimum Dimensions and Choice of Form to R.C. Cast-In-Situ Prismatic Roofs. IASS Symposium, vol. 2, Vienna.

(78) Hahn, L. (1970). A Roof Structure of Folded Plates (Conference Hall of the Unesco Building in Paris. IASS Symposium, vol. 2, Vienna.

(79) Amsler, Th. A. (1970). Alleghany Airlines Hangar, Logan International Airport, Boston, Mass., U.S.A. IASS Symposium, vol. 2, Vienna.

(80) Wilby, C. B. (1998). Concrete folded plate roofs. Arnold, London, p. 12.

(81) www.ketchum.org/-milo/what.html (visita 10 enero de 2010).

(82) Our Lady of the Assumption Parish Church. Canadian architect, 53(12): 44-46. 2008. 